\title{
Maternal protein restriction with or without folic acid supplementation during pregnancy alters the hepatic transcriptome in adult male rats
}

\author{
Karen A. Lillycrop ${ }^{1}$, Joanne Rodford ${ }^{2}$, Emma S. Garratt ${ }^{2}$, Joanne L. Slater-Jefferies ${ }^{2}$, Keith M. Godfrey ${ }^{3}$, \\ Peter D. Gluckman ${ }^{4}$, Mark A. Hanson ${ }^{2}$ and Graham C. Burdge ${ }^{2}$ \\ ${ }^{1}$ Development and Cell Biology, Biomedical Sciences Building, University of Southampton, Bassett Crescent East, Southampton \\ SO16 7PX, UK \\ ${ }^{2}$ Institute of Developmental Sciences, Southampton General Hospital, Tremona Road, Southampton SO16 6YD, UK \\ ${ }^{3}$ Medical Research Council Epidemiology Resource Centre, Southampton General Hospital, Tremona Road, Southampton SO16 \\ $6 Y D, U K$ \\ ${ }^{4}$ Liggins Institute, University of Auckland, Private Bag 92019, Auckland, New Zealand
}

(Received 4 August 2009 - Revised 9 December 2009 - Accepted 10 December 2009 - First published online 9 March 2010)

Feeding pregnant rats a protein-restricted (PR) diet induces altered expression of candidate genes in the liver of the adult offspring, which can be prevented by supplementation of the PR diet with folic acid (PRF). We investigated the effect of maternal nutrition during pregnancy on the liver transcriptome in their adult male offspring. Pregnant rats were fed control, PR or PRF diets. Male offspring were killed on day 84. The liver transcriptome was analysed by microarray (six livers per maternal dietary group) followed by post hoc analysis of relative mRNA levels and gene ontology. These results were confirmed for selected genes by real-time RT-PCR. There were 311 genes that differed significantly ( $\geq 1.5$-fold change; $P<0.05$ ) between PR offspring (222 increased) and control offspring, while 191 genes differed significantly between PRF offspring (forty-five increased) compared with offspring of control dams. There were sixteen genes that were significantly altered in both PR and PRF offspring compared with controls. Ion transport, developmental process, and response to reactive oxygen species (RROS) and steroid hormone response (SHR) ontologies were altered in PR offspring. Folic acid supplementation prevented changes within RROS and SHR response pathways, but not in ion transport or developmental process. There was no effect of maternal PR on mRNA expression of imprinted genes. Insulin 1 and Pleckstrin homology-like domain family A member 2 were increased significantly in PRF compared with PR offspring. The present findings show that the pattern of induced changes in the adult liver transcriptome were dependent on maternal protein and folic acid intakes during pregnancy.

Transcriptome: Maternal diet: Microarray analysis: Protein restriction: Folate

Environmental cues acting through developmental plasticity induce altered expression of the genome which, in turn, changes the phenotype of the offspring ${ }^{(1)}$. In human subjects a poor in utero environment is associated with reduced prenatal growth and increased risk of chronic diseases including cardiometabolic disease in later life ${ }^{(2)}$. These findings have been replicated in animal models where restricted nutrition during pregnancy induces dyslipidaemia, obesity, hypertension, hyerinsulinaemia and hyperleptinaemia in the offspring ${ }^{(3,4)}$.

The mechanism by which cues about nutrient availability in the prenatal environment are transmitted to the fetus and the process by which different phenotypes are induced are poorly understood. However, induction of an altered phenotype in the offspring that persists throughout the lifespan implies stable changes to gene transcription which then result in altered activities of metabolic pathways and homeostatic control processes ${ }^{(5)}$. A number of studies in animal models have demonstrated that poor maternal nutrition during pregnancy does indeed induce persistent changes in gene expression in the offspring. Feeding pregnant rats a protein-restricted (PR) diet during pregnancy induces increased expression of the glucocorticoid receptor (GR) in the liver, lung, kidney and brain of the offspring during fetal, neonatal and adult life ${ }^{(6-8)}$ which can be transmitted, at least in liver, to a second generation ${ }^{(9)}$. In the liver, increased GR expression is associated with increased expression of its target gene phosphoenolpyruvate carboxykinase $^{(7,8)}$ and up-regulation of gluconeogenesis ${ }^{(10)}$. Expression of PPAR- $\alpha$ is also increased in the liver of offspring born to dams fed a PR diet during pregnancy and is accompanied by the increased expression of its target gene acyl-CoA oxidase which is rate limiting in peroxisomal $\beta$-oxidation ${ }^{(7)}$. However, increasing the folic acid content of the maternal $\mathrm{PR}$ diet prevented altered expression of PPAR $\alpha$ and $\mathrm{GR}^{(7)}$.

Abbreviations: GR, glucocorticoid receptor; Ins1, insulin 1; Phlda2, Pleckstrin homology-like domain family A member 2; PR, protein restricted; PRF, protein-restricted diet with folic acid.

* Corresponding author: Dr K. A. Lillycrop, fax +44 2380 594459, email k.a.lillycrop@soton.ac.uk 
In contrast, $70 \%$ global undernutrition reduced hepatic PPAR $\alpha$ and GR expression in the liver of the adult offspring $^{(11)}$, which suggests that different nutritional exposures during pregnancy induce different phenotypes in the offspring. This is consistent with the hypothesis that the fetus adapts to environmental cues in a manner which predicts the future environment, thus potentially conferring a Darwinian fitness advantage ${ }^{(12)}$. Other examples of persistent changes in gene expression induced by poor maternal diet during pregnancy include $11 \beta$-hydroxysteroid dehydrogenase-2, glucokinase, fatty acid synthase, acetyl-CoA carboxylase, angiotensin $1 \mathrm{~b}$ and 2 receptors and carnitine palmitoyl transferase- $1^{(6,13-15)}$. For at least some of the genes which show altered mRNA expression in response to poor prenatal nutrition, altered transcriptional activity has been shown to be associated with changes in their epigenetic regulation by DNA methylation and/or histone modifications ${ }^{(7,8,14,16)}$, although there are exceptions such as glucokinase ${ }^{(13)}$.

To date, studies on long-term changes in gene expression induced by maternal protein restriction have used a candidate gene approach. While this has produced useful findings, this approach does not allow assessment of the specificity of the changes induced by maternal dietary constraint in the transcriptome of the offspring or which gene networks are affected. We have used a genome-wide microarray to determine the specificity of the effect of differences in maternal nutrition during pregnancy on gene expression in their offspring. We investigated the effect of feeding pregnant rats a PR diet or the PR diet supplemented with folic acid on hepatic gene expression in their adult male offspring.

\section{Materials and methods}

\section{Animals and tissues}

All animal procedures were carried out in accordance with the Home Office Animals (Scientific Procedures) Act (1986). The tissues reported here were from the same animals as described previously ${ }^{(17)}$. Virgin female Wistar rats (about $250 \mathrm{~g}$ ) (six rats per diet) were mated and fed one of three diets from conception until delivery (each group contained six females): control, a PR diet, or the PR diet supplemented with additional folic acid (PRF) (Table 1). Diets were manufactured by Special Diets Services (Witham, Essex, UK). Dams were allowed to deliver spontaneously at about $21 \mathrm{~d}$ and litters were reduced to eight rats, equal males and females, within $24 \mathrm{~h}$ after birth. Dams were fed the standard semi-purified American Institute of Nutrition (AIN)-76G during lactation. Offspring were weaned at $28 \mathrm{~d}$ onto a nutritionally adequate diet and were killed by asphyxiation with $\mathrm{CO}_{2}$ after food had been withdrawn for $6 \mathrm{~h}$ on postnatal day 84 . Livers were removed immediately, frozen in liquid $\mathrm{N}_{2}$ and stored at $-80^{\circ} \mathrm{C}$. Six livers from male offspring, one per litter, were selected in each dietary group for analysis. One additional liver from a male offspring of a control dam was collected as the reference for the microarray analysis.

\section{RNA isolation and measurement of the expression of the liver transcription by Agilent oligonucleotide array hybridisation}

RNA was extracted from each liver using the SV Total RNA Extraction Kit (Promega, Madison, WI, USA). RNA was quantified by absorbance at $260 \mathrm{~nm}$ and the integrity of the $28 \mathrm{~s}$ and $18 \mathrm{~s}$ ribosomal RNA was verified by agarose gel electrophoresis. In all cases the absorbance ratio at 260 and $280 \mathrm{~nm}$ was greater than 2. Microarray hybridisation and analysis was carried out by Oxford Gene Technology (OGT, Oxford, UK) in accordance with the company's qualitycontrol procedures using standard protocols for labelling, hybridisation and washing (Two Colour Microarray Based Gene expression analysis version; Agilent Technologies, Inc., Palo Alto, CA, USA). Six microarrays were performed per dietary group. Briefly, total RNA (300 ng) extracted from the liver of each rat was transcribed into cDNA. After denaturation of the RT enzyme, samples were then transcribed

Table 1. Composition of diets fed to pregnant and lactating dams, and to the offspring after weaning

\begin{tabular}{|c|c|c|c|c|c|}
\hline & \multicolumn{3}{|c|}{ Diet fed during pregnancy } & \multirow[b]{2}{*}{ Diet fed during lactation: AIN-76A } & \multirow[b]{2}{*}{ Post-weaning diet } \\
\hline & Control & PR & PRF & & \\
\hline Casein $(\mathrm{g} / \mathrm{kg})$ & 180 & 90 & 90 & 200 & 180 \\
\hline Folic acid (mg/kg) & 1 & 1 & 5 & 2 & 1 \\
\hline Maize starch (g/kg) & 425 & 485 & 485 & 150 & 455 \\
\hline Sucrose $(\mathrm{g} / \mathrm{kg})$ & 213 & 243 & 243 & 500 & 243 \\
\hline Choline $(\mathrm{g} / \mathrm{kg})$ & 2 & 2 & 2 & 2 & 2 \\
\hline Methionine $(\mathrm{g} / \mathrm{kg})$ & 5 & 5 & 5 & 3 & 5 \\
\hline Vitamin mix $(\mathrm{g} / \mathrm{kg})^{\star}$ & 5 & 5 & 5 & 5 & 5 \\
\hline Mineral mix $(\mathrm{g} / \mathrm{kg}) \dagger$ & 20 & 20 & 20 & 20 & 20 \\
\hline Cellulose $(\mathrm{g} / \mathrm{kg})$ & 50 & 50 & 50 & 50 & 50 \\
\hline Maize oil $(\mathrm{g} / \mathrm{kg})$ & 0 & 0 & 0 & 50 & 0 \\
\hline Soyabean oil (g/kg) & 100 & 100 & 100 & 0 & 4 \\
\hline Lard $(g / k g)$ & 0 & 0 & 0 & 0 & 36 \\
\hline Total metabolisable energy (MJ/kg) & $17 \cdot 3$ & $17 \cdot 5$ & $17 \cdot 5$ & $15 \cdot 5$ & $16 \cdot 1$ \\
\hline
\end{tabular}

PR, protein restricted; PRF, protein restricted with folic acid; AIN, American Institute of Nutrition.

${ }^{*}$ Vitamin mix: thiamin hydrochloride, $2.4 \mathrm{mg} / \mathrm{kg}$; riboflavin, $2.4 \mathrm{mg} / \mathrm{kg}$; pyridoxine hydrochloride, $2.8 \mathrm{mg} / \mathrm{kg} ;$ nicotinic acid, $12.0 \mathrm{mg} / \mathrm{kg} ; \mathrm{D}$-calcium pantothenate, $6.4 \mathrm{mg} / \mathrm{kg}$; biotin, $0.01 \mathrm{mg} / \mathrm{kg}$; cyanocobalamin, $0.003 \mathrm{mg} / \mathrm{kg}$; retinyl palmitate, $6.4 \mathrm{mg} / \mathrm{kg} ;$ DL- $\alpha$-tocopheryl acetate, $79.9 \mathrm{mg} / \mathrm{kg} ;$ cholecalciferol, $1.0 \mathrm{~g} / \mathrm{kg}$; menaquinone, $0.02 \mathrm{mg} / \mathrm{kg}$.

† Mineral mix: calcium phosphate dibasic, $11.3 \mathrm{~g} / \mathrm{kg}$; sodium chloride, $1.7 \mathrm{~g} / \mathrm{kg}$; potassium citrate monohydrate, $5.0 \mathrm{~g} / \mathrm{kg} ;$ potassium sulfate, $1.2 \mathrm{~g} / \mathrm{kg}$; magnesium sulfate, $0.5 \mathrm{~g} / \mathrm{kg}$; magnesium carbonate, $0.1 \mathrm{~g} / \mathrm{kg}$; ferric citrate, $0.1 \mathrm{~g} / \mathrm{kg}$; zinc carbonate, $36.2 \mathrm{mg} / \mathrm{kg}$; cupric carbonate, $6.8 \mathrm{mg} / \mathrm{kg}$; potassium iodate, $0.2 \mathrm{mg} / \mathrm{kg}$; sodium selenite, $0.2 \mathrm{mg} / \mathrm{kg}$; chromium potassium sulfate, $12.5 \mathrm{mg} / \mathrm{kg}$. 
into cRNA and labelled with the fluorescent dye Cy (test sample Cy3, reference sample Cy5). Each test sample together with a reference sample (prepared from an additional control animal) was hybridised to an Agilent 014879 whole rat genome array $(4 \times 44 \mathrm{~K}) \mathrm{G} 4131 \mathrm{~F}$. This array contains 45018 features with 41012 unique probes. Microarray slides were scanned at $5 \mu \mathrm{m}$ resolution using the extended dynamic range (Hi $100 \%$, Low $10 \%$ ). The slides were then feature extracted using Agilent feature extraction software 9.5.3.1. All arrays were uploaded into Genespring GX V 7.3 (Silicon Genetics Inc., Palo Alto, CA, USA) for data normalisation, quality control and first-pass analysis. All arrays were normalised per spot and per chip using an intensity-dependent normalisation (Lowess normalisation) using Genespring (http://stat-www.berkeley.edu/users/terry/zarray/Html/normspie. html). This is a within-slide normalisation that adjusts for intensity-dependent variation due to dye properties. The expression ratios were calculated for each probe by dividing the Cy3 processed signal by Cy5 processed signal. The identification of the genes showing increased or decreased expression was performed using GeneSifter $^{\mathrm{TM}}$ software (www.genesifter.net; VizX Labs LLC, Seattle, WA, USA) by averaging the expression ratios from the six samples and running a Wilcoxon rank sum test (significance set at $P<0.05)$ with a Benjamini and Hochberg false discovery rate correction.

\section{Ontology report}

Gene ontology reports (Biological Process and Molecular Function), based on the Gene Ontology Consortium (http:// www.geneontology.org/GO.doc.html) ${ }^{(18)}$, including $z$-score analyses, were generated using GeneSifter ${ }^{\mathrm{TM}}$ software. A $z$-score is a statistical rating of the relative expression of gene ontologies and indicates how much each ontology is over-represented (positive $z$-score) or under-represented (negative $z$-score) in a gene list. Positive $z$-scores $(>2)$ reflect gene ontology categories with a greater number of genes meeting the criterion than is expected by chance, while negative $z$-scores $(>-2)$ identify gene ontology categories with a lower number of genes meeting the criterion by chance.

\section{Real-time RT-PCR}

To verify the changes in gene expression detected by microarray analysis, we analysed the expression of six genes - TNF $\alpha$, inducible NO synthase, c-Fos, NFкB, uncoupling protein (UCP)-3 and thyroid receptor (TR)-1 $\beta$ - which differed in microarray analysis between control and PR offspring. Liver samples (approximately $100 \mathrm{mg}$ ) were pulverised under liquid $\mathrm{N}_{2}$ and the resulting powder was divided and used to prepare mRNA ${ }^{(8)}$. mRNA expression was measured by real-time RT-PCR ${ }^{(8)}$. Briefly, total RNA was isolated from cells with TRIzol reagent (Invitrogen, Paisley, UK), and $1 \mu \mathrm{g}$ was used as a template to prepare cDNA with 100 units of Moloney murine leukaemia virus RT. cDNA was amplified with real-time RT-PCR primers specific for the products of the TNF $\alpha$, inducible NO synthase, c-Fos, NFkB, uncoupling protein 3 and TR-1 $\beta$ (Table 2). The reaction was performed in a total volume of $25 \mu$ l with SYBR Green Jumpstart Ready Mix (Sigma, Poole, Dorset, UK).

\section{Statistical methods}

From the microarray data the identification of the genes showing increased or decreased expression was performed using GeneSifter ${ }^{\mathrm{TM}}$ software (www.genesifter.net; VizX Labs LLC) by averaging the expression ratios from six samples and running a Wilcoxon rank sum test (significance set at $P<0.05)$ with a Benjamini and Hochberg false discovery rate correction. For RT-PCR verification of the expression array data statistical significance was determined using a one-way ANOVA with a Bonferroni's post hoc analysis.

\section{Results \\ Effects of maternal dietary exposure on the hepatic transcriptome of the offspring}

The mRNA expression of 311 genes differed by $\geq 1 \cdot 5$-fold $(P<0.05)$ between PR and control offspring. The expression of 222 genes was increased in the PR offspring compared with control offspring, while eighty-nine genes were downregulated (Supplemental Table 1). The twenty genes which showed the greatest difference in expression between PR offspring and controls are shown in Table 3.

The mRNA expression of 191 genes was significantly different $(\geq 1.5$-fold; $P<0.05)$ between $P R F$ and control offspring. Of these, forty-five genes showed higher expression and 146 genes showed lower expression compared with controls (Table 4 and Supplemental Table 2). Only sixteen of the 311 genes which differed significantly between PR and control offspring differed significantly between PRF and control offspring (Table 5).

There were 480 genes that differed significantly ( $\geq 1 \cdot 5$-fold; $P<0.05)$ between PRF and PR offspring. Of these, 463 genes showed greater expression and 230 genes showed lower expression in PRF than PR offspring (Table 6 and

Table 2. Primers for analysis of mRNA expression by real-time RT-PCR

\begin{tabular}{lcc}
\hline Gene & Forward primer & Reverse primer \\
\hline C-Fos & 5'-GGCAAAGTAGAGCAGCTATCTCCT-3' $^{\prime}$ & 5'-TCAGCTCCCTCCTCCGATTC-3' $^{\prime}$ \\
iNOS & 5'-CAGCGGGATGACTTTCCAAG-3' $^{\prime}$ 5'-AGGCAAGATTTGGACCTGCA-3' $^{\prime}$ \\
TNF $\alpha$ & QuantiTect Primer Assay QT00178717 \\
NFkB & QuantiTect Primer Assay QT01577975 \\
TR-1 $\beta$ & QuantiTect Primer Assay QT00193690 \\
UCP3 & $5^{\prime}$-CTGGACTCTCACCTGTTCAC-3' & $5^{\prime}$-GCCACCATCCTCAGCATAC-3'
\end{tabular}

iNOS, inducible NO synthase; TR-1 $\beta$, thyroid receptor $1 \beta$; UCP3, uncoupling protein 3. 
Table 3. Genes with higher or lower expression (top twenty) in liver of the offspring of dams fed a proteinrestricted (PR) diet compared with controls*

\begin{tabular}{llll}
\hline Gene name & Gene identifier & Ratio & Direction \\
\hline UDP glucuronosyltransferase precursor & J02589 & $26 \cdot 94$ & Down \\
Interferon-inducible GTPase & NM_001024884 & $20 \cdot 71$ & Up \\
UDP glycosyltransferase 2 family, polypeptide B & NM_031533 & $17 \cdot 28$ & Down \\
Cyclin B3 & XM_228779 & $4 \cdot 53$ & Down \\
Olfactory receptor 1326 & NM_001000474 & $3 \cdot 89$ & Down \\
Unknown & TC558741 & $3 \cdot 64$ & Down \\
Unknown & TC563179 & $3 \cdot 61$ & Down \\
Unknown & XM_223906 & $3 \cdot 50$ & Up \\
Nebulin & XM_229925 & $3 \cdot 38$ & Up \\
Periostin, osteoblast specific factor & XM_342245 & $3 \cdot 37$ & Up \\
Solute carrier family 13 (sodium/sulfate symporters), member 1 NM_031651 & $3 \cdot 33$ & Down \\
Centaurin, $\alpha 1$ & NM_133567 & $3 \cdot 29$ & Up \\
Signal peptide, CUB domain, EGF-like 3 & XM_228030 & $3 \cdot 25$ & Down \\
Gap junction membrane channel protein $\alpha 7$ & XM_001081521 & $3 \cdot 20$ & Up \\
3-Oxoacid CoA transferase 1 & XM_001073523 & $3 \cdot 14$ & Up \\
Fc receptor, IgE, low affinity II, $\alpha$ polypeptide & NM_133550 & $3 \cdot 11$ & Up \\
Unknown & AW142860 & $3 \cdot 08$ & Down \\
Mucin 13 & Al044457 & $3 \cdot 02$ & Down \\
Adenylate kinase 5 isoform 1 & XM_001080050 & $3 \cdot 01$ & Up \\
Chemokine (C-X-C motif) ligand 14 & NM_001013137 & $3 \cdot 00$ & Down \\
\hline
\end{tabular}

* The ratio is calculated as the average expression ratio between the offspring of dams fed a PR $(n 6)$ or a control $(n 6)$ diet Direction denotes whether the change in expression between PR and control offspring is increased or decreased. All genes showed a significant change in expression between PR and control offspring $(P<0.05)$. $P$ values were calculated using the Wilcoxon ranking feature with the Benjamini and Hochberg correction.

Supplemental Table 3). Of the 311 genes whose expression was significantly altered in PR compared with control offspring, ninety-two were also significantly altered in PRF compared with control offspring (Supplemental Table 4).

\section{Pathway analysis by gene ontology}

Data were categorised on two independent gene ontology terms, Biological Process and Molecular Function. In the Biological Processes ontology, the major pathways affected in the PR offspring compared with control offspring ( $z$-score greater than $2 ; P>0.05)$ among the up-regulated genes were ion transport, developmental process and response to stress, in particular response to oxidative stress (Fig. 1). For the down-regulated genes, response to steroid hormone stimulus was significantly over-represented. In PRF $v$. control offspring, the pathways significantly over-represented were ion transport, developmental process, fatty acid and steroid metabolic process amongst the up-regulated genes. No pathways were significantly affected amongst the down-regulated genes.

Table 4. Genes that were up- or down-regulated (top twenty) in the liver of the offspring of dams fed a protein-restricted diet supplemented with folic acid (PRF) compared with controls*

\begin{tabular}{|c|c|c|c|}
\hline Gene name & Gene identifier & Ratio & Direction \\
\hline UDP glucuronosyltransferase precursor, gene & J02589 & $22 \cdot 61$ & Down \\
\hline UDP glycosyltransferase 2 family, polypeptide B & NM 031533 & 11.35 & Down \\
\hline Casein kinase II, $\alpha 1$ & BF551036 & 8.13 & Down \\
\hline Anterior pharynx defective 1a homolog & NM_001014255 & 5.92 & Down \\
\hline E74-like factor 2 (Elf2), transcript variant 2 & NM_001012181 & $5 \cdot 18$ & Down \\
\hline Copine IV & XM_001070003 & 4.54 & Down \\
\hline RIKEN cDNA 4932415L06 gene & XM_237289 & 3.94 & Down \\
\hline Chemokine (C-C motif) ligand 11 & NM_019205 & 3.72 & Down \\
\hline Class VI alcohol dehydrogenase & S79716 & 3.53 & Down \\
\hline Olfactory receptor 1337 & NM_001000480 & 3.51 & Down \\
\hline Small nuclear ribonucleoprotein D3 & XM_001079870 & 3.26 & Down \\
\hline Carnitine acetyltransferase & NM_001004085 & 3.19 & Up \\
\hline Growth hormone 1 & NM_001034848 & 2.93 & Down \\
\hline Sema domain, transmembrane domain (TM), and cytoplasmic domain, (semaphorin) $6 \mathrm{~A}$ & XM 341612 & $2 \cdot 91$ & Down \\
\hline B-box and SPRY domain containing & NM_022261 & $2 \cdot 89$ & Up \\
\hline Zinc finger, MYND domain containing 19 & NM_198770 & $2 \cdot 88$ & Down \\
\hline Unknown & BF552300 & $2 \cdot 84$ & Down \\
\hline Unknown & BQ196294 & $2 \cdot 79$ & Down \\
\hline Unknown [BF550568] & BF550568 & 2.68 & Up \\
\hline Jumonji domain containing 4 & XM_220561 & 2.58 & Down \\
\hline
\end{tabular}

\footnotetext{
* The ratio is calculated as the average ratio in expression levels between the offspring of dams fed a PRF diet $(n 6)$ and controls $(n 6)$. Direction denotes whether the change in expression between PRF and control offspring is increased or decreased. All genes showed a significant change in expression between PRF and control offspring $(P<0 \cdot 05)$. $P$ values were calculated using the Wilcoxon ranking feature with the Benjamini and Hochberg correction.
} 
Table 5. Genes significantly altered in the liver of the offspring of dams fed a protein-restricted (PR) diet and protein-restricted diet supplemented with folic acid (PRF) compared with a control diet ${ }^{*}$

\begin{tabular}{|c|c|c|c|c|c|}
\hline Gene name & Gene identifier & PR v. control ratio & Direction & PRF $v$. control ratio & Direction \\
\hline UDP glucuronosyltransferase precursor & J02589 & 26.94 & Down & $22 \cdot 61$ & Down \\
\hline UDP glycosyltransferase 2 family, polypeptide B & NM_031533 & $17 \cdot 28$ & Down & $11 \cdot 35$ & Down \\
\hline Jumonji domain containing 4 & XM_220561 & $1 \cdot 8$ & Down & 2.58 & Down \\
\hline Leucine rich repeat containing 2 & XM_001056081 & 1.96 & Down & 2.52 & Down \\
\hline Thyroid hormone receptor $\beta$ & NM_012672 & 2.51 & Down & 2.51 & Down \\
\hline Unknown & AW142860 & 3.08 & Down & $2 \cdot 22$ & Down \\
\hline Olfactory receptor 276 & NM_001000227 & 1.99 & Down & $2 \cdot 14$ & Down \\
\hline Butyrylcholinesterase & NM_022942 & 1.77 & Down & 1.92 & Down \\
\hline Rous sarcoma oncogene & NM_031977 & 1.9 & Down & 1.91 & Down \\
\hline Unknown & DV728362 & 1.54 & Down & 1.72 & Down \\
\hline KIAA0157 gene product & XM_219441 & 1.88 & Down & $1 \cdot 71$ & Down \\
\hline Natural cytotoxicity triggering receptor 3 & NM_181822 & 1.91 & Down & $1 \cdot 68$ & Down \\
\hline Transmembrane glycoprotein A33 antigen & XM_341149 & $2 \cdot 37$ & Up & 1.67 & Up \\
\hline Tudor domain containing 3 & NM_001012043 & 1.61 & Down & 1.64 & Down \\
\hline Solute carrier family 4 , member 4 & NM_053424 & 1.75 & Down & 1.59 & Down \\
\hline Unknown & XM_573395 & 1.76 & Down & 1.59 & Down \\
\hline
\end{tabular}

* The ratio is calculated as the average ratio in expression levels between either the offspring of dams fed a PR diet ( $n$ 6) and the control diet ( $n 6)$, or between offspring of dams fed a PRF diet $(n 6)$ and the control diet $(n 6)$. Direction indicates whether the change in expression between PR or PRF and control offspring is increased or decreased. All genes showed a significant change in expression between PR and control offspring and between PRF and control offspring $(P<0.05)$. $P$ values were calculated using the Wilcoxon ranking feature with the Benjamini and Hochberg correction.

Comparison of PRF $v$. PR offspring revealed that the major pathways affected amongst the over-expressed genes were response to steroid hormone, steroid metabolic process and fatty acid metabolic process and among the down-regulated genes response to oxidative stress.

In the Molecular Function ontology, the major categories altered among the up-regulated genes in the PR $v$. control offspring were receptor binding, tetrapyrrole binding, and cation transmembrane transporter activity and among the down-regulated genes pathways affected were UDPglycosyltransferase activity, anion transmembrane transporter activity, growth factor activity and ATPase activity (Fig. 2). No pathways were over-represented in PRF offspring compared with controls. In PRF $v$. PR offspring, receptor binding, oxidoreductase activity, transcription factor cofactor activity were among the up-regulated genes and acetyl transferase activity and tetrapyrrole binding were among the down-regulated genes.

\section{Imprinted genes}

There was no effect of maternal protein restriction on mRNA expression of imprinted genes, although insulin-1 (InsI)

Table 6. Genes that were up- or down-regulated (top twenty) in the liver of the offspring of dams fed a protein-restricted diet supplemented with folic acid (PRF) compared with offspring of dams fed a protein-restricted (PR) diet $^{\star}$

\begin{tabular}{llcc}
\hline Gene name & Gene identifier & Ratio & Direction \\
\hline RT1 class lb & AW142896 & $11 \cdot 86$ & Down \\
Camello-like 3 & XM_575585 & $5 \cdot 75$ & Down \\
Cyclin B3 & XM_228779 & $4 \cdot 9$ & Up \\
X-linked gene & NM_019203 & $4 \cdot 68$ & Up \\
ATPase, H ${ }^{+}$transporting, V0 subunit D, isoform 2 & NM_001011972 & $4 \cdot 57$ & Down \\
Q96AL3 PHA4 protein & TC522828 & $4 \cdot 27$ & Up \\
Unknown & TC525504 & $3 \cdot 95$ & Up \\
Similar to Eph receptor A4 & XM_244186 & $3 \cdot 9$ & Up \\
Unknown & TC558494 & $3 \cdot 77$ & Up \\
Copine IV & XM_001070003 & $3 \cdot 74$ & Down \\
Phosphorylase kinase $\gamma 1$ & NM_031573 & $3 \cdot 38$ & Down \\
Unknown & TC557723 & $3 \cdot 29$ & Down \\
Chemokine (C-C motif) ligand 11 & NM_019205 & $3 \cdot 21$ & Down \\
N-acetyltransferase 8 & NM_022635 & $3 \cdot 2$ & Down \\
Unknown & TC544566 & $3 \cdot 18$ & Down \\
Protein tyrosine phosphatase, receptor type, J & NM_017269 & $3 \cdot 16$ & Up \\
Hypothetical protein FLJ10901 & XM_213984 & $3 \cdot 14$ & Up \\
Unknown & TC560462 & $3 \cdot 11$ & Down \\
Rho/rac guanine nucleotide exchange factor (GEF) 18 & XM_221775 & $3 \cdot 11$ & Up \\
RNAJ3232 mRNA for MHC class II RT1-D 31 chain haplotype k & AJ003232 & $3 \cdot 1$ & Up \\
\hline
\end{tabular}

* The ratio is calculated as the average ratio in expression levels between the offspring of PRF $(n 6)$ and PR offspring $(n 6)$ dams. Direction indicates whether the change in expression between PRF and PR offspring is increased or decreased. All genes showed a significant change in expression between PRF and PR offspring $(P<0.05)$. $P$ values were calculated using the Wilcoxon ranking feature with the Benjamini and Hochberg correction. 

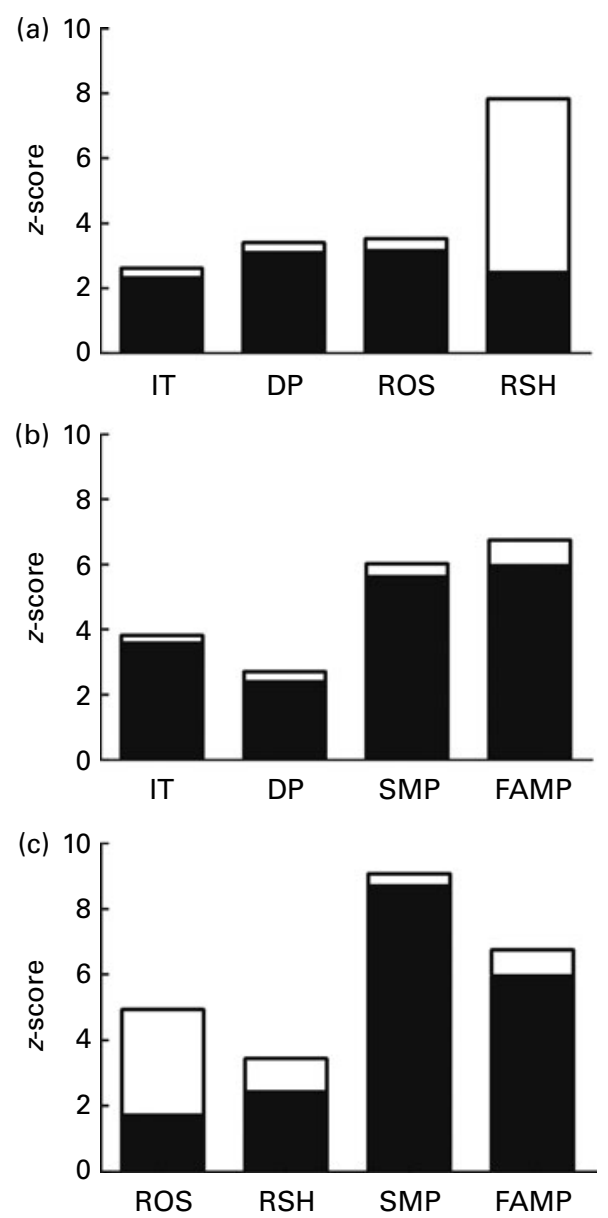

Fig. 1. Pathways over-represented in the gene ontology Biological Processes category. Values are based on six offspring in each of the maternal dietary groups: control, protein-restricted (PR) and PR supplemented with folic acid (PRF). (a) Control v. PR; (b) control v. PRF; (c) PR v. PRF. Pathways with $z$-scores above 2 are plotted for both up-regulated genes (匚) and downregulated $(\square)$ genes. IT, ion transport; DP, developmental processes; ROS, response to oxidative stress; RSH, response to steroid hormone; SMP, steroid metabolic processes; FAMP, fatty acid metabolic processes.

(2-2-fold increase) and Pleckstrin homology-like domain family A member 2 (Phlda2) (1.7-fold increase) were significantly altered in PRF compared with PR offspring (Supplemental Table 3).

\section{Validation of array by real-time RT-PCR}

The mRNA expression of TNF $\alpha$, iNos, c-Fos, NFkB, uncoupling protein 3 and thyroid receptor- $1 \beta$ agreed with the corresponding data from the array (Fig. 3).

\section{Discussion}

Maternal dietary protein restriction during pregnancy has been reported to induce long-term changes in the expression of a number of candidate genes within the offspring ${ }^{(5)}$. However, the range of genes reported to date reflects the interests of the researchers and does not provide an indication of the proportion of the transcriptome which is affected or which gene networks and pathways show the greatest
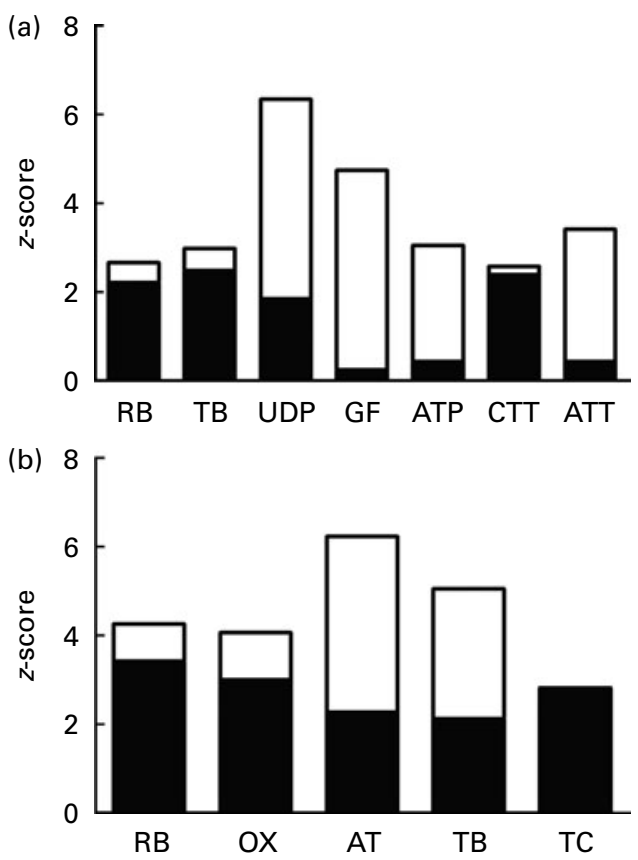

Fig. 2. Pathways over-represented in the gene ontology Molecular Function category. Values are based on six offspring in each of the maternal dietary groups: control, protein-restricted (PR) and PR supplemented with folic acid (PRF). (a) Control v. PR; (b) PR v. PRF. Pathways with $z$-scores above 2 are plotted for both up-regulated genes $(\square)$ and down-regulated $(\square)$ genes. $\mathrm{RB}$, receptor binding; TB, tetrapyrrole binding; UDP, UDP-glycosyltransferase activity; GF, growth factor activity; ATP, ATPase activity; CTT, cation transporter activity; ATT, anion transporter activity; OX, oxidoreductase activity; AT, acetyl transferase activity; TC, transcription cofactor activity.

or least susceptibility. The present results show for the first time that a small proportion of genes are affected in adult liver by variation in the protein and folic acid content of the maternal diet. The microarray allows measurement of the mRNA expression of approximately 39000 transcripts (Agilent). Based on this estimate, maternal dietary protein restriction during pregnancy induced the altered expression of approximately $1.3 \%$ of the liver transcriptome which was reduced to $0.7 \%$ by increasing the amount of folic acid in the maternal PR diet. These data suggest that as the changes in gene expression are limited to a relatively small subset of genes within the genome this may reflect an adaptive response induced in response to maternal protein restriction. A similar proportion of genes was reported to have significantly changed in the placenta (E17.5) of mice in response to maternal protein restriction ${ }^{(19)}$. Genes with a wide range of functions were found to be altered in PR $v$. control offspring but this is consistent with the studies to date which have shown that offspring from PR dams show a number of the metabolic and physiological alterations including increased fat deposition and altered feeding behaviour ${ }^{(20-22)}$, impaired glucose homeostasis $^{(10)}$, vascular dysfunction ${ }^{(20,23)}$, impaired immunity ${ }^{(24)}$ and increased susceptibility to oxidative stress $^{(25)}$ and tumorigenesis $^{(26)}$. For instance, although the genes showing the greatest change in expression between control and PR offspring have not previously been demonstrated to be changed in response to maternal diet, changes in their expression is consistent with some of the phenotypic changes reported 


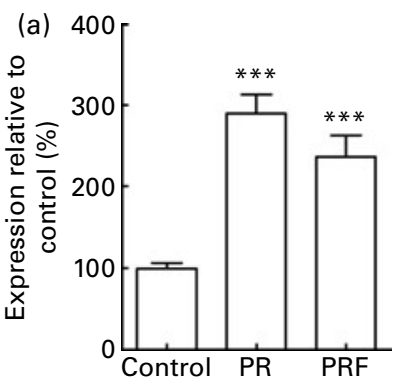

Maternal dietary group

(c) 300

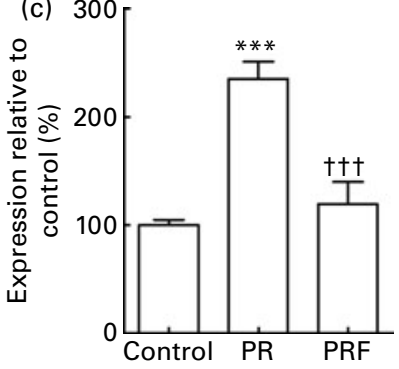

Maternal dietary group

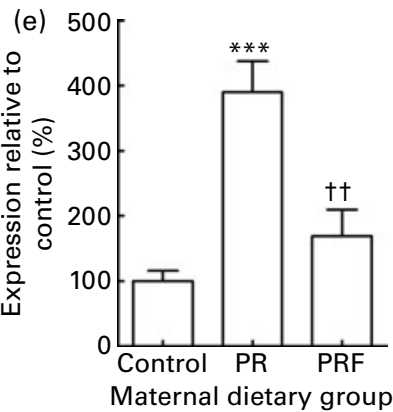

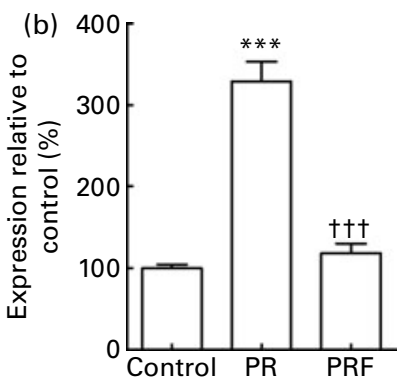

Maternal dietary group

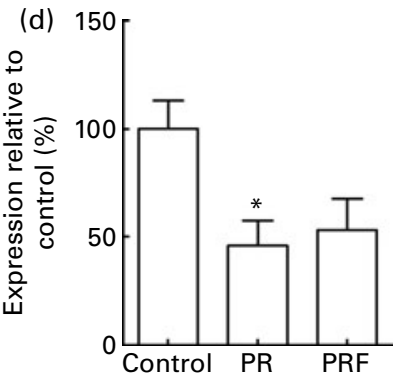

Maternal dietary group

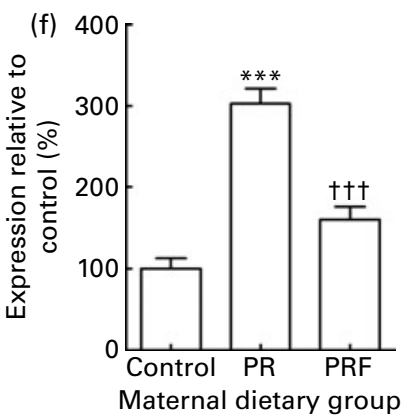

Fig. 3. mRNA expression of c-Fos (a), inducible NO synthase (iNOS) (b), TNF $\alpha$ (c), thyroid receptor $1 \beta($ TR-1 $1 \beta)$ (d), NFKB (e) and uncoupling protein (UCP)-3 (f) in liver from day 84 male offspring of dams fed either a control, protein-restricted (PR) or PR supplemented with folic acid (PRF) diet during pregnancy. Values are means for six offspring, with standard errors represented by vertical bars. Statistical comparisons are by one-way ANOVA with Bonferroni's post hoc analysis. Only statistically significant differences are shown. Mean value was significantly different from that of the control group: ${ }^{\star} P<0.05,{ }^{* \star \star} P<0.0001$. Mean value was significantly different from that of the PR group: $\dagger \dagger P<0.01$, $\dagger+\dagger P<0.0001$. Overall probabilities were C-FOs, $P<0.0001$; iNOS, $P<0.0001$; TNF $\alpha, P<0.0001$; TR-1 $\beta, P=0.022$; NFКB, $P=0.002$; UCP3, $P<0.0001$.

to be induced in PR offspring. UDP-glucuronosyltransferase precursor, which shows a $26 \cdot 94$-fold decrease in expression in PR offspring, plays a major role in conjugation and subsequent elimination of potentially toxic xenobiotics and endogenous compounds. Decreased expression and function of this enzyme have been linked to a number of human cancers $^{(27-29)}$. Thus, this decrease in PR offspring may in part explain the increased susceptibility of PR offspring to carcinogenesis $^{(26)}$. Interferon- $\gamma$-induced GTPase showed a 20 -fold increase in expression in PR offspring. Interferon- $\gamma$ GTPase is important for host immunological defence ${ }^{(30)}$ which is consistent with an increased tendency towards inflammation in PR offspring ${ }^{(31)}$. However, it may be that not all of the changes in gene expression have functional consequences; some of the changes may simply reflect the process of adaptation. For instance, the olfactory receptor 1326 is up-regulated in PR offspring. Olfactory receptors are known to play roles in odour sensing ${ }^{(32)}$ and in environmental sensing in non-olfactory tissues including the kidney ${ }^{(33)}$. Thus, although their role in any adaptive process in this nutritional model is unclear, they may play an as-yet uncharacterised role in regulating cell function which may contribute to the induced phenotype.

Previous studies have shown that supplementation of the maternal PR diet with folic acid or the methyl donor glycine prevents the induction of hypertension, dyslipidaemia and altered hepatic PPAR $\alpha$ and GR expression in the offspring ${ }^{(7,34,35)}$. The present findings are in part consistent with these studies. We also compared gene expression changes in PRF offspring as well. We found that, while maternal protein restriction induced the altered expression of $1.3 \%$ of the genome, this was reduced to $0.7 \%$ of the genome in PRF compared with control offspring. However, in the present study only $7 \%$ of genes which differed in expression between control and PRF offspring also differed between control and PR offspring. Within this set of genes altered in response to both maternal PR and PRF, we found that the maternal PR diet tended to induce increased gene expression while the PRF diet reversed this trend. Together, these findings indicate that although increasing the folic acid content of the maternal PR diet prevented some of the effects on the transcriptome induced by the PR diet alone, increasing the level of folic acid relative to protein did not simply correct for the PR diet. This is consistent with the finding that the maternal PRF diet decreased weight gain in the offspring, which suggests that the apparently beneficial effect of increased folic acid intake on some metabolic processes may be at the expense of others ${ }^{(17)}$. Supplementation of the diet of the offspring of dams fed control or PR diets with folic acid after weaning induced specific changes in the mRNA expression of specific genes and in fat metabolism ${ }^{(35)}$. Furthermore, while the maternal PRF diet prevented hypomethylation of specific $\mathrm{CpG}$ dinucleotides in the liver PPAR $\alpha$ promoter, it also increased the methylation level of two others which suggests a shift, albeit modest, in gene control ${ }^{(16)}$.

Ontology analysis showed altered maternal diet induced different changes in individual pathways in the liver of the adult offspring depending on the nature of the maternal diet. Comparison of control $v$. PR offspring showed that ion transport, developmental process, and response to steroid hormone and reactive oxygen species were the pathways significantly altered. Alterations in these pathways are consistent with previous phenotypic studies which show that ion transport $^{(36)}$, cell commitment in blastocysts ${ }^{(37)}$, response to reactive oxygen species $^{(25)}$ and steroid hormones ${ }^{(6,38)}$ are altered by maternal diet. Response to steroid hormone and reactive oxygen species pathways were not altered in PRF offspring, suggesting that folate supplementation corrected these changes induced by PR. However, ion transport and developmental process pathways remained altered in PRF offspring, demonstrating that folate supplementation does not reverse all gene expression changes induced by protein restriction. Interestingly we also observed that fatty acid and steroid metabolic process pathways were altered in PRF offspring but not in PR offspring, suggesting that folate 
supplementation induces distinct changes in gene expression. Thus, the results of these analyses are consistent with the findings of measurements of individual genes in that although the PRF diet prevented changes in gene expression in some pathways, it either did not affect others or changed the expression of genes in pathways not affected by the PR diet. This is in contrast to previous studies which show that for a limited number of phenotypic outcomes increasing the folic acid content of the PR diet prevents the induction of an altered phenotype $^{(17,23)}$.

Changes in the epigenetic regulation of imprinted genes results in a range of developmental abnormalities and changes in metabolic processes in humans ${ }^{(39-41)}$ and agricultural animals ${ }^{(42)}$, and in some animal models of metabolic disease ${ }^{(43)}$. It has been proposed that altered regulation of imprinted genes changes the partitioning of nutrient resources and so may represent one mechanism for the induction of impaired nutrient homeostasis in the offspring by prenatal environmental constraint ${ }^{(44)}$. There were no differences in the expression in the liver of imprinted genes between control and PR offspring, although the imprinted genes Ins 1 and Phlda2 were significantly altered in PRF compared with PR offspring. This suggests that induction of an altered phenotype in the offspring by modest variations in maternal protein restriction does not involve changes in imprinted genes. This is consistent with the differences in the patterns of disease associated with prenatal environmental constraint and those associated with impaired imprinting ${ }^{(39,45)}$. However, interestingly, folate supplementation did alter the expression of Ins1 and Phlda2. Folate supplementation of patients with hyperhomocysteinaemia has also been reported to alter the methylation of imprinted genes ${ }^{(46)}$, suggesting that imprinted genes may be susceptible to changes in dietary folate intake.

Together, the present findings show that the long-term regulation of the transcriptome reflects the nature of the prenatal nutritional environment. It has been suggested that phenotypes induced by variations in the intra-uterine environment may have adaptive value ${ }^{(12)}$. Whether the induced changes in this relatively small subset of genes are adaptive or not requires further experimentation, including more detailed characterisation of the induced phenotype which accompanies such alterations in mRNA levels.

\section{Acknowledgements}

The study was funded in part by a fellowship to G. C. B. from the British Heart Foundation (FS/05/064/19525), who also provide salary support to M. A. H., and by the EpiGen consortium which funded the microarray analysis.

G. C. B., K. A. L., P. D. G. and M. A. H. designed the study. K. A. L., J. R., E. S. G. and J. S.-J. carried out the laboratory procedures and analysed the data. K. A. L. and G. C. B. wrote the manuscript with input from all authors.

None of the authors had any financial or personal conflicts of interest.

The supplementary material (Supplemental Tables 1-4) for this article can be found at http://www.journals. cambridge.org/bjn

\section{References}

1. Bateson P, Barker D, Clutton-Brock T, et al. (2004) Developmental plasticity and human health. Nature 430, 419-421.

2. Godfrey KM \& Barker DJ (2001) Fetal programming and adult health. Public Health Nutr 4, 611-624.

3. Bertram CE \& Hanson MA (2001) Animal models and programming of the metabolic syndrome. Br Med Bull 60, $103-121$.

4. Armitage JA, Taylor PD \& Poston L (2005) Experimental models of developmental programming: consequences of exposure to an energy rich diet during development. J Physiol 565, 3-8.

5. Burdge GC, Hanson MA, Slater-Jefferies JL, et al. (2007) Epigenetic regulation of transcription: a mechanism for inducing variations in phenotype (fetal programming) by differences in nutrition during early life? $B r J$ Nutr 97, 1036-1046.

6. Bertram C, Trowern AR, Copin N, et al. (2001) The maternal diet during pregnancy programs altered expression of the glucocorticoid receptor and type $211 \beta$-hydroxysteroid dehydrogenase: potential molecular mechanisms underlying the programming of hypertension in utero. Endocrinology 142, $2841-2853$

7. Lillycrop KA, Phillips ES, Jackson AA, et al. (2005) Dietary protein restriction of pregnant rats induces and folic acid supplementation prevents epigenetic modification of hepatic gene expression in the offspring. J Nutr 135, 1382-1386.

8. Lillycrop KA, Slater-Jefferies JL, Hanson MA, et al. (2007) Induction of altered epigenetic regulation of the hepatic glucocorticoid receptor in the offspring of rats fed a proteinrestricted diet during pregnancy suggests that reduced DNA methyltransferase- 1 expression is involved in impaired DNA methylation and changes in histone modifications. $\mathrm{Br} J \mathrm{Nutr}$ 97, 1064-1073.

9. Burdge GC, Slater-Jefferies J, Torrens C, et al. (2007) Dietary protein restriction of pregnant rats in the $\mathrm{F} 0$ generation induces altered methylation of hepatic gene promoters in the adult male offspring in the F1 and F2 generations. Br J Nutr 97, 435-439.

10. Burns SP, Desai M, Cohen RD, et al. (1997) Gluconeogenesis, glucose handling, and structural changes in livers of the adult offspring of rats partially deprived of protein during pregnancy and lactation. J Clin Invest 100, 1768-1774.

11. Gluckman PD, Lillycrop KA, Vickers MH, et al. (2007) Metabolic plasticity during mammalian development is directionally dependent on early nutritional status. Proc Natl Acad Sci U S A 104, 12796-12800.

12. Gluckman PD \& Hanson MA (2004) Living with the past: evolution, development, and patterns of disease. Science $\mathbf{3 0 5}$, 1733-1736.

13. Bogdarina I, Murphy HC, Burns SP, et al. (2004) Investigation of the role of epigenetic modification of the rat glucokinase gene in fetal programming. Life Sci 74, 1407-1415.

14. Bogdarina I, Welham S, King PJ, et al. (2007) Epigenetic modification of the renin-angiotensin system in the fetal programming of hypertension. Circ Res 100, 520-526.

15. Maloney CA, Gosby AK, Phuyal JL, et al. (2003) Site-specific changes in the expression of fat-partitioning genes in weanling rats exposed to a low-protein diet in utero. Obes Res 11, 461-468.

16. Lillycrop KA, Phillips ES, Torrens C, et al. (2008) Feeding pregnant rats a protein-restricted diet persistently alters the methylation of specific cytosines in the hepatic PPAR $\alpha$ promoter of the offspring. Br J Nutr 100, 278-282.

17. Burdge GC, Lillycrop KA, Jackson AA, et al. (2008) The nature of the growth pattern and of the metabolic response to fasting in the rat are dependent upon the dietary protein and folic acid intakes of their pregnant dams and post-weaning fat consumption. Br J Nutr 99, 540-549. 
18. Ashburner M, Ball CA, Blake JA, et al. (2000) Gene ontology: tool for the unification of biology. The Gene Ontology Consortium. Nat Genet 25, 25-29.

19. Gheorghe CP, Goyal R, Holweger JD, et al. (2009) Placental gene expression responses to maternal protein restriction in the mouse. Placenta 30, 411-417.

20. Lucas A, Baker BA, Desai M, et al. (1996) Nutrition in pregnant or lactating rats programs lipid metabolism in the offspring. Br J Nutr 76, 605-612.

21. Bellinger L, Lilley C \& Langley-Evans SC (2004) Prenatal exposure to a maternal low-protein diet programmes a preference for high-fat foods in the young adult rat. Br J Nutr 92, 513-520.

22. Bellinger L, Sculley DV \& Langley-Evans SC (2006) Exposure to undernutrition in fetal life determines fat distribution, locomotor activity and food intake in ageing rats. Int $J$ Obes (Lond) 30, 729-738.

23. Torrens C, Brawley L, Anthony FW, et al. (2006) Folate supplementation during pregnancy improves offspring cardiovascular dysfunction induced by protein restriction. Hypertension 47, 982-987.

24. Calder PC \& Yaqoob P (2000) The level of protein and type of fat in the diet of pregnant rats both affect lymphocyte function in the offspring. Nutr Res 20, 995-1005.

25. Langley-Evans SC \& Sculley DV (2005) Programming of hepatic antioxidant capacity and oxidative injury in the ageing rat. Mech Ageing Dev 126, 804-812.

26. Fernandez-Twinn DS, Ekizoglou S, Gusterson BA, et al. (2007) Compensatory mammary growth following protein restriction during pregnancy and lactation increases earlyonset mammary tumor incidence in rats. Carcinogenesis $\mathbf{2 8}$, $545-552$.

27. McGrath M, Lepine J, Lee IM, et al. (2009) Genetic variations in UGT1A1 and UGT2B7 and endometrial cancer risk. Pharmacogenet Genomics 19, 239-243.

28. Mitra PS, Basu NK \& Owens IS (2009) Src supports UDPglucuronosyltransferase-2B7 detoxification of catechol estrogens associated with breast cancer. Biochem Biophys Res Commun 382, 651-656.

29. Cecchin E, Innocenti F, D'Andrea M, et al. (2009) Predictive role of the UGT1A1, UGT1A7, and UGT1A9 genetic variants and their haplotypes on the outcome of metastatic colorectal cancer patients treated with fluorouracil, leucovorin, and irinotecan. J Clin Oncol 27, 2457-2465.

30. Henry SC, Daniell XG, Burroughs AR, et al. (2009) Balance of Irgm protein activities determines IFN- $\gamma$-induced host defense. J Leukoc Biol 85, 877-885.

31. Calder PC (2009) Early life programming of immune and lung function: can we now exclude a role of arachidonic acid exposure? Br J Nutr 102, 331-333.
32. Zhao H \& Firestein S (1999) Vertebrate odorant receptors. Cell Mol Life Sci 56, 647-659.

33. Pluznick JL, Zou DJ, Zhang X, et al. (2009) Functiona expression of the olfactory signaling system in the kidney. Proc Natl Acad Sci U S A 106, 2059-2064.

34. Jackson AA, Dunn RL, Marchand MC, et al. (2002) Increased systolic blood pressure in rats induced by a maternal low-protein diet is reversed by dietary supplementation with glycine. Clin Sci (Lond) 103, 633-639.

35. Burdge GC, Lillycrop KA, Phillips ES, et al. (2009) Folic acid supplementation during the juvenile-pubertal period in rats modifies the phenotype and epigenotype induced by prenatal nutrition. J Nutr 139, 1054-1060.

36. Jansson T \& Powell TL (2000) Placental nutrient transfer and fetal growth. Nutrition 16, 500-502.

37. Kwong WY, Wild AE, Roberts P, et al. (2000) Maternal undernutrition during the preimplantation period of rat development causes blastocyst abnormalities and programming of postnatal hypertension. Development 127, 4195-4202.

38. Langley-Evans SC, Phillips GJ, Benediktsson R, et al. (1996) Protein intake in pregnancy, placental glucocorticoid metabolism and the programming of hypertension in the rat. Placenta 17, 169-172.

39. Temple IK (2007) Imprinting in human disease with special reference to transient neonatal diabetes and BeckwithWiedemann syndrome. Endocr Dev 12, 113-123.

40. Amor DJ \& Halliday J (2008) A review of known imprinting syndromes and their association with assisted reproduction technologies. Hum Reprod 23, 2826-2834.

41. Guo L, Choufani S, Ferreira J, et al. (2008) Altered gene expression and methylation of the human chromosome 11 imprinted region in small for gestational age (SGA) placentae. Dev Biol 320, 79-91.

42. Sinclair KD, Young LE, Wilmut I, et al. (2000) In-utero overgrowth in ruminants following embryo culture: lessons from mice and a warning to men. Hum Reprod 15, Suppl. 5, 68-86.

43. Shao WJ, Tao LY, Gao C, et al. (2008) Alterations in methylation and expression levels of imprinted genes H19 and Igf2 in the fetuses of diabetic mice. Comp Med 58, 341-346.

44. Charalambous M, da Rocha ST \& Ferguson-Smith AC (2007) Genomic imprinting, growth control and the allocation of nutritional resources: consequences for postnatal life. Curr Opin Endocrinol Diabetes Obes 14, 3-12.

45. Gluckman PD \& Hanson MA (2006) The consequences of being born small - an adaptive perspective. Horm Res $\mathbf{6 5}$ Suppl. 3, 5-14.

46. Ingrosso D, Cimmino A, Perna AF, et al. (2003) Folate treatment and unbalanced methylation and changes of allelic expression induced by hyperhomocysteinaemia in patients with uraemia. Lancet 361, 1693-1699. 